Article

\title{
Tactile Feels in Grasping/Cutting Processes with Scissors
}

\author{
Mioto Waga ${ }^{1}$, Yuuki Aita ${ }^{1}$, Junichi Noma ${ }^{2}$ and Yoshimune Nonomura ${ }^{1, *}$ \\ 1 Department of Biochemical Engineering, Graduate School of Science and Engineering, Yamagata University, \\ 4-3-16 Jonan, Yonezawa, Yamagata 992-8510, Japan; tyc55948@st.yamagata-u.ac.jp (M.W.); \\ tde88827@st.yamagata-u.ac.jp (Y.A.) \\ 2 Kurimoto Ltd., Suminoe, Osaka 559-0021, Japan; j_noma@kurimoto.co.jp \\ * Correspondence: nonoy@yz.yamagata-u.ac.jp; Tel.: +81-238-26-3164
}

Received: 20 June 2018; Accepted: 11 July 2018; Published: 14 July 2018

check for updates

\begin{abstract}
Understanding the dynamic phenomena in grasping/cutting processes with scissors is important for the design of surgical robots and virtual reality systems. Here, we show the relationship between the mechanical stimuli and tactile sensations when forceps or scissors are used. Nineteen subjects grasped or cut objects and evaluated the tactile sensations in each of the processes. To conduct the tactile and mechanical evaluation simultaneously, subjects operated scissors that were fixed to a mechanical evaluation system. When subjects grasped urethane resin, stainless steel plate, and adhesive tape, soft, hard, and sticky feels were perceived, respectively. Dry, hard, and creaking feels were perceived in the paper cutting process. In addition, we observed four characteristic tangential force profiles in the processes. Regression analysis suggests the following findings: Hardness is perceived by the change of force and blade movement when the scissors make contact with the object. Stickiness is caused by the increase and decrease of force at the moment of peeling when the scissors break contact with the object. The cutting sensation is affected by fine force fluctuations during the scissors closing and the rapidly decreased force at the moment of cutting completion.
\end{abstract}

Keywords: scissors; tactile feels; mechanical evaluation

\section{Introduction}

Scissors are tools that grasp or cut objects by a sandwiching action. In many cases, the object is grasped or cut by closing two elongated metal plates, which are overlapped at the fulcrum. This tool contains a leverage structure consisting of two blades at the action point, a connecting part at the fulcrum, and a handle at the point of force. Scissor-type instruments were developed in the period between 1300 before Christ (BC) to $600 \mathrm{BC}$ [1]. Today, scissors are indispensable tools and are used in a variety of fields, including grooming, gardening, textiles manufacturing, and medicine. Recently, scissors have been mounted on surgical robots and precision working robots to grasp and cut by remote control [2]. In such systems, operators manipulate without feedback of tactile information, which typically contributes to determining the amplitude of the force applied to objects [3-5]. A understanding of the dynamic phenomena during grasping/cutting processes is required to develop haptic display systems or simulation systems.

Many attempts have been made to model mechanical phenomena in the cutting process of objects. Mahvash et al. and Tanaka et al. have proposed computing methods to show the mechanical phenomena when objects are cut vertically by a single blade [6,7]. Greenish et al. measured the forces involved when living tissues were cut with three kinds of scissors and constructed a database [8]. Mahvash et al. calculated the cutting forces involved from the contact conditions of blade to object. Additionally, they verified the accuracy of the model using experimental data obtained from cutting 
paper or chicken [9]. Okamura et al. modeled the cutting force from the standpoints of friction, motion, and physical properties of objects [10]. Fujino et al. calculated the force applied to the hand, the restoring force of the leaf spring portion, and the friction inside the device when a test subject cut selected objects with micro shearing blades [11]. Weiss et al. developed two-dimensional, deformable, mass-spring models. They simulated the deformation of the tissue mesh from the position, speed, net force, and external force vector of each node on the virtual blade [12]. Fratu et al. calculated the forces applied to the scissors from a torque-angle response model synthesized from measurement data multiplied by a ratio that depends on the position of the cutting crack edge and the curve of the blades. The calculated forces were displayed by a 2 degree-of-freedom haptic scissors system [13]. Funahashi et al. displayed cutting sensation by combining vibrations and cutting sound [14].

Scissors and forceps are used not only for cutting, but also for grasping objects. In this study, the mechanical stimuli were measured when a test subject grasped or cut an object with scissors. The objects were selected to analyze the relationship between tactile feels and physical stimuli. Nineteen subjects evaluated the tactile feels when they grasped or cut objects with different moduli of elasticity or different surface properties. Urethane resin, stainless steel plate, and adhesive tape were grasped with forceps to clarify the physical origin of the hardness and stickiness. To clarify the physical origin of the cutting sensation, the paper was cut with scissors. The mechanical stimuli and movements of the scissors during the grasping and cutting processes were measured using a mechanical evaluation system equipped with a high-speed camera and strain gauges [15]. We analyzed the relationship between the tactile feels and the dominant physical parameters.

\section{Materials and Methods}

\subsection{Scissors and Objects}

The forceps (total length: $160 \mathrm{~mm}$ ) and scissors $(80 \mathrm{~mm}$ ) were obtained from Globeride Co., Ltd. (Tokyo, Japan) and PIP Co., Ltd. (Osaka, Japan), respectively (Figure 1). Objects for the grasping test were as follows: Urethane resin (thickness: $10 \mathrm{~mm}$; human skin gel concentrate, Asker C = 15, Exseal Co., Ltd., Gifu, Japan) was covered with a polytetrafluoroethylene seat (thickness: $0.1 \mathrm{~mm}$, Nichias Co., Ltd., Tokyo, Japan). The Young's modulus of the covered resin was $0.72 \pm 0.08 \mathrm{MPa}$ (average \pm standard deviation). Nine stainless steel plates (thickness: $1 \mathrm{~mm}$; SUS304) were stacked to obtain a $9 \mathrm{~mm}$-thick stainless steel block. Adhesive tape (thickness: $1.1 \mathrm{~mm}$; 3M Japan Holdings G.K., Tokyo, Japan) was wrapped around an acrylic plate (thickness: $5 \mathrm{~mm}$; Mitsubishi rayon Co., Ltd., Tokyo, Japan). Paper (thickness: 0.3 mm; APP Japan Co., Ltd., Tokyo, Japan) was selected as an object for the cutting test.

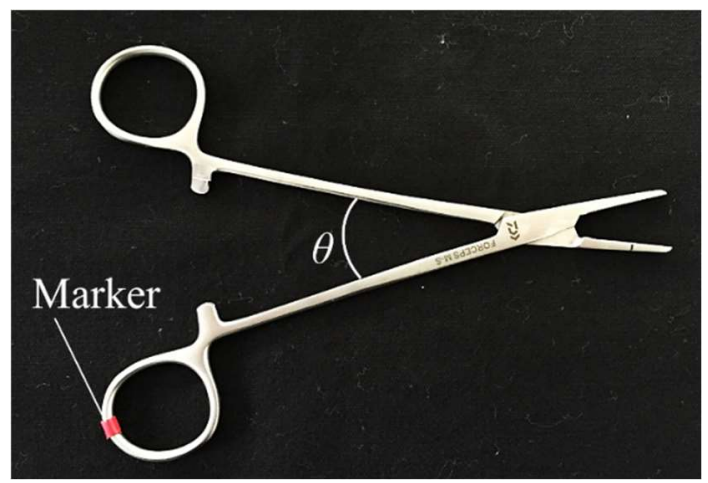

(a)

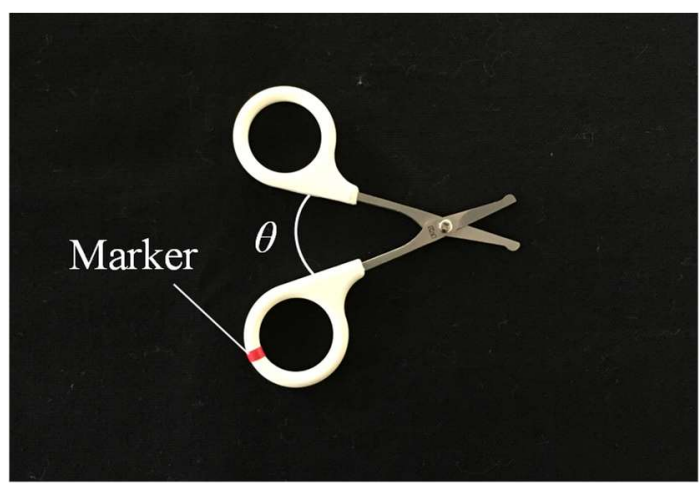

(b)

Figure 1. Photograph of the (a) forceps and (b) scissors. 


\subsection{Tactile Evaluation Tests}

We carried out tactile evaluations to observe the relationship between the physical properties of the objects and the sensations experienced by the test subjects during the grasping and cutting processes. Simultaneously, we carried out mechanical evaluations using a mechanical evaluation system with a high-speed camera. The evaluations were conducted in a quiet room at a temperature of $25 \pm 1{ }^{\circ} \mathrm{C}$ and the relative humidity was $50 \pm 5 \%$. The test subjects consisted of 9 males and 10 females aged 21-25 years old. Prior to the evaluations, the test subjects washed both hands with commercial liquid hand-soap. One handle of the scissors was fixed to the mechanical evaluation system and test subjects were instructed to grip the other handle and to repeat the opening and closing motion for twenty seconds parallel to the tangential direction of the system (Figure 2). A marker was put on the suitable position on a handle to fix the gripping position for all of the subjects (Figure 1). The objects for the grasping and cutting tests were fixed at a position $10 \mathrm{~mm}$ from the tip of the scissors. After releasing their fingers from the scissors, subjects completed questionnaires about their sensations during test. Each of the subjects rated the tactile feels during the grasping and cutting processes: The most applicable position on a $100 \mathrm{~mm}$ line with opposite descriptions at each end was marked. The descriptive pairings were as follows: "Soft" /"not soft," "hard" / "not hard," "sticky" /"not sticky," "snapped" /"not snapped," "smooth" /"not smooth," "creaking" / "not creaking," and "dry" /"not dry", which were selected by preliminary evaluation. For example, if a mark is placed $30 \mathrm{~mm}$ away from one end written as "not soft", the score of "soft" is 3.0. The second question was "please describe freely about tactile feels when you manipulated scissors." The test objects were presented in a random order to eliminate the order effect, while the identity of all objects was not revealed to the subjects. In addition, the subjects touched the scissors through a blackout curtain to exclude the effects of sight. All evaluations adhered to the principles expressed in the Declaration Helsinki. A responsible party at Yamagata University confirmed that the ethics and safety of the present test were acceptable.

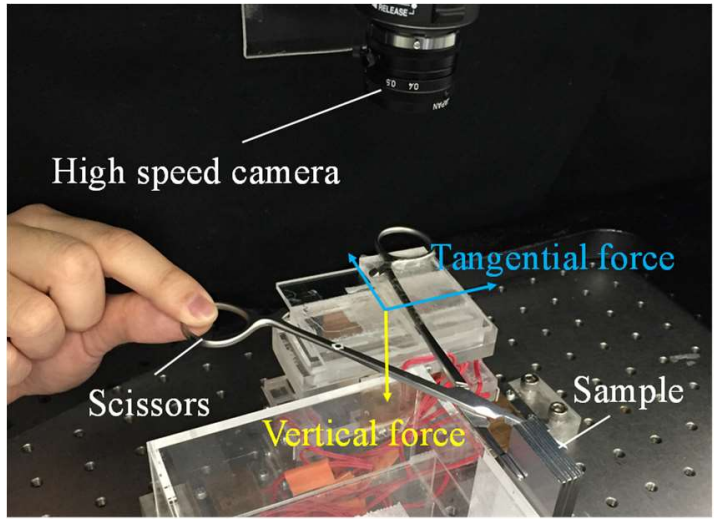

(a)

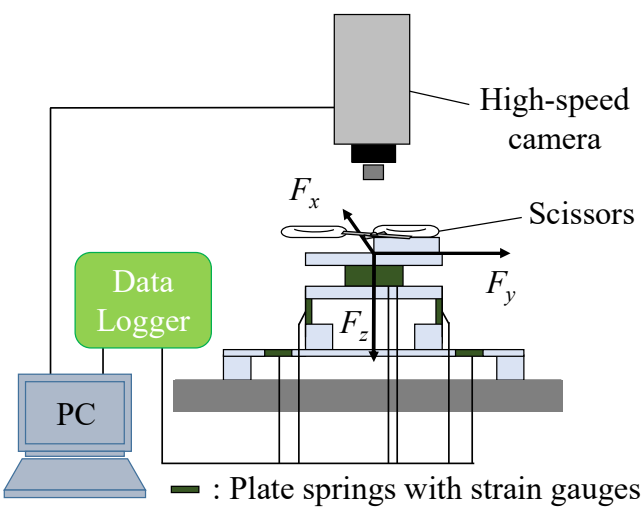

(b)

Figure 2. (a) A photograph and (b) diagram of the mechanical evaluation system used for the simultaneous measurement of the motion and force properties during grasping/cutting processes.

\subsection{Mechanical Evaluation Tests}

We evaluated the tactile feels, mechanical force, and movement of the blades using the mechanical evaluation system simultaneously (Figure 2). Since one handle of the scissors was fixed to the mechanical evaluation system, the force could be regarded as equal to the reaction force applied to the hand. We calculated the tangential resultant force, $F_{x y}$, using following equation:

$$
F_{x y}=\sqrt{F_{x}^{2}+F_{y}^{2}}
$$


here, $F_{x}$ and $F_{y}$ are the tangential force applied to the $x$ and $y$ direction of the evaluation system. Tangential and vertical forces were monitored using strain gauges (KFG-03-120-C1-11 M2R, Kyowa Electronic Instruments Co., Ltd., Tokyo, Japan) on leaf springs. Three pairs of leaf springs were placed at the device's $x, y$, and $z$ directions, respectively. These strain gauges were connected to a personal computer (PC) (VW9000, Keyence Co., Tokyo, Japan) through a data logger unit composed of two electronic measurement systems (NR-500 and NR-ST04, Keyence Co., Tokyo, Japan). The detection limits of the $F_{x}$ and $F_{y}$, and the vertical force $\left(F_{z}\right)$ were $2.9 \times 10^{-3}, 2.0 \times 10^{-4}$, and $3.9 \times 10^{-2} \mathrm{~N}$, respectively. The ranges of the linear responses for $F_{x}, F_{y}$, and $F_{z}$ were $8.8 \times 10^{-3}$ to $9.8 \mathrm{~N}, 2.9 \times 10^{-4}$ to $9.8 \mathrm{~N}$, and $5.9 \times 10^{-2}$ to $11.8 \mathrm{~N}$, respectively. During the evaluation, we obtained high-speed images from just above the scissors with a high-speed camera (VW-300M, Keyence Co., Tokyo, Japan). Data from the mechanical evaluation and the high-speed observations were acquired at $2 \mathrm{~ms}$ intervals. The captured images were analyzed using the Motion Analyzer VW-H2MA analysis software (Keyence Co., Tokyo Japan) to obtain the angle, $\theta$, between the handles.

\section{Results}

\subsection{Tactile Evaluation Tests}

Figure 3 shows the score that test subjects rated the tactile feels of the grasping/cutting processes. The scores were dependent on the physical properties of the objects. When the test subjects grasped the urethane resin, soft, smooth, and dry feels were strongly perceived: The scores were $8.0 \pm 2.6$, $6.2 \pm 3.1$, and $6.0 \pm 3.3$, respectively. In the case of stainless steel plates, hard and dry feels were perceived: The scores were $7.1 \pm 3.9$ and $7.6 \pm 3.2$, respectively. For the adhesive tape, sticky and hard feels were reported, with scores of $8.4 \pm 2.6$ and $6.4 \pm 2.7$, respectively. On the other hand, when the subjects cut paper, creaking, hard, dry, and snapped feels were perceived: The scores were $6.6 \pm 2.8,6.8 \pm 2.5,8.2 \pm 2.7$, and $4.3 \pm 3.5$, respectively. These results show that the subjects felt soft, hard, or sticky feels when they grasped urethane resin, stainless plate, and adhesive tape, respectively. In addition, the creaking and dry feels were strongly perceived when they cut paper without sight. Hard feel was not only recognized in the process of grasping a stainless steel plate or adhesive tape wrapped around an acrylic plate, but also in the process of cutting paper.

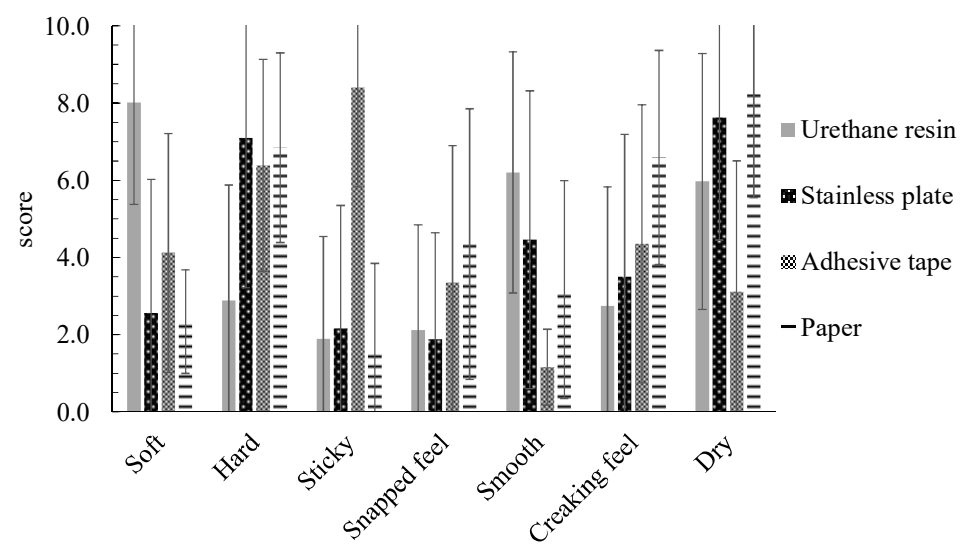

Figure 3. Tactile scores during grasping or cutting of objects with scissors.

\subsection{Mechanical Evaluation Tests}

In the grasping/cutting processes with scissors, significant differences were observed in the profiles of $F_{x y}$. They were classified into four patterns qualitatively by neglecting small differences. Figure 4 shows the typical temporal profiles of $F_{x y}$ and $\theta$ in a single operation. The temporal zero point was adjusted to the time at which the edge of the scissors touched the objects. The characteristics of each profile are as follows: 
1. One-step increase pattern: As shown in Figure 4a, when subjects grasped the soft urethane resin, a pattern in which $F_{x y}$ increased slowly was observed in many cases. We defined this profile as a "one-step increase pattern." From the moment when the blade touched the resin, the tangential force increased substantially with a constant slope. At $0.5 \mathrm{~s}$, the force attained $3.8 \mathrm{~N}$. At the start of the grasping process, $\theta$ was $18^{\circ}$. Then, as the force achieved maximum value, the $\theta$ decreased to $6^{\circ}$;

2. Two-step increase pattern: As shown in Figure $4 \mathrm{~b}$, when a hard stainless plate was grasped by the forceps, a pattern in which $F_{x y}$ increased through a two-step process was observed in many cases. The slope was mild in the second step, while it was steep in the first step. We defined this profile as a "two-step increase pattern". After the resultant force increased to $1.9 \mathrm{~N}$ at $0.06 \mathrm{~s}$, the force increased to $2.3 \mathrm{~N}$ at $0.5 \mathrm{~s}$. At the start of the grasping process, $\theta$ was $17^{\circ}$; then, as the force achieved the maximum value, $\theta$ decreased to $16^{\circ}$;

3. Double peak pattern: A characteristic profile was obtained when the adhesive tape was grasped (Figure 4c). We defined this profile as a "double peak pattern." A peak, which was caused by the peeling of the tape from the blade, was observed during the handle opening. $F_{x y}$ increased to $0.8 \mathrm{~N}$ at $0.65 \mathrm{~s}$ in the process of opening the handle and decreased to $0.1 \mathrm{~N}$ at the moment when the tape was peeled from the blade (at $0.75 \mathrm{~s}$ ); and

4. Drastic decrease pattern: When a subject cut the paper, the $F_{x y}$ decreased sharply and then increased again to reach the maximum value (Figure $4 \mathrm{~d}$ ). We defined this profile as a "drastic decrease pattern." On average, the cutting process began when the $\theta$ was $13^{\circ}$. The resultant force fluctuated (amplitude of $0.07 \pm 0.05 \mathrm{~N}$ ), increased to $0.7 \mathrm{~N}$ at $0.2 \mathrm{~s}$, and then decreased to $0.3 \mathrm{~N}$ at the moment the cutting was completed (when $\theta$ was $1^{\circ}$ ). After that, the $\theta$ decreased to $0^{\circ}$ and the force became $1.3 \mathrm{~N}$ at $0.3 \mathrm{~s}$. During the cutting process, the $F_{x y}$ fluctuated finely due to the friction among the scissor components and the paper.

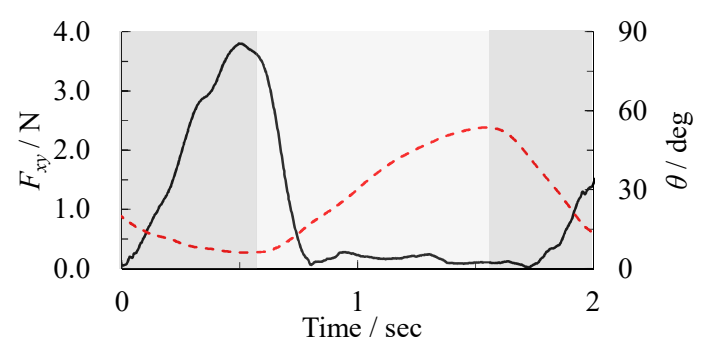

(a)

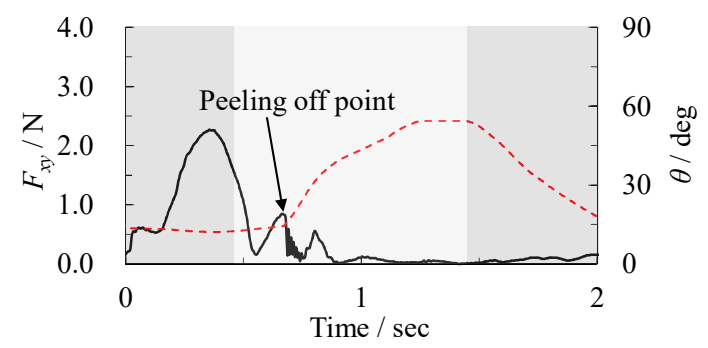

(c)

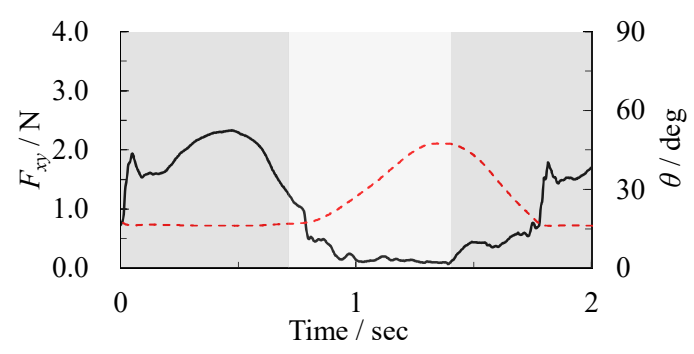

(b)

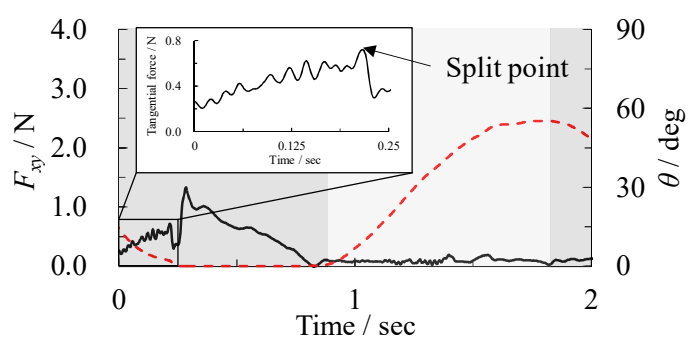

(d)

Figure 4. Temporal profiles of tangential resultant force $F_{x y}$ (straight lines) and the angle between the handles $\theta$ (dotted-lines) over time as a subject (a) grasped the urethane resin (one-step increase pattern), (b) the stainless steel plate (two-step increase pattern), (c) the adhesive tape (double peak pattern), and (d) cut the paper (drastic decrease pattern). Dark gray zones indicate the closing phase and light gray zones indicate the opening phase. 


\subsection{Relationship Between the Tactile and Mechanical Evaluations}

We calculated the incidence rates of the four patterns: The number of appearing times of the one-step increase pattern, two-step increase pattern, and double peak pattern in the grasping processes were counted to determine the incidence rates. The incidence rates of the drastic decrease pattern in the cutting process were calculated. Figure 5 shows the relationship between the incidence rates of each profile and the score of some tactile feels, including soft, hard, sticky, and snapped feels. The incidence rates of the one-step increase pattern and soft feel indicated a positive correlation (correlation coefficient $R=0.541$ ), while that of the two-step increase pattern and hard feel showed a positive correlation $(R=0.538)$. The incidence rates of the double peak pattern and sticky feel showed a positive correlation $(R=0.760)$. The drastic decrease pattern and snapped feel showed a positive correlation $(R=0.485)$.

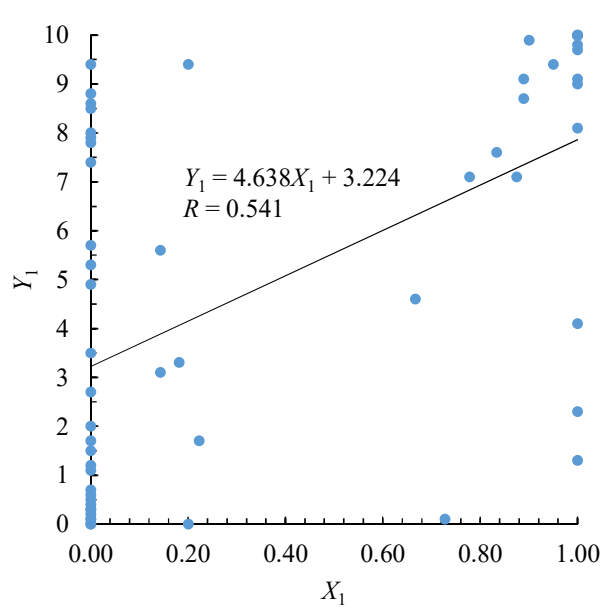

(a)

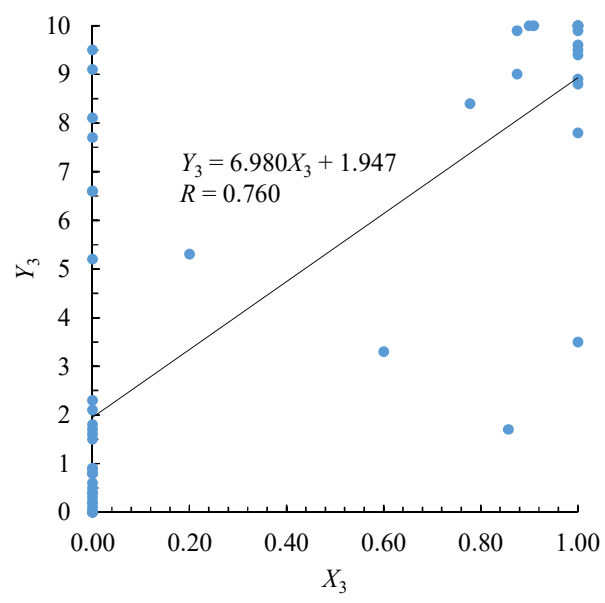

(c)

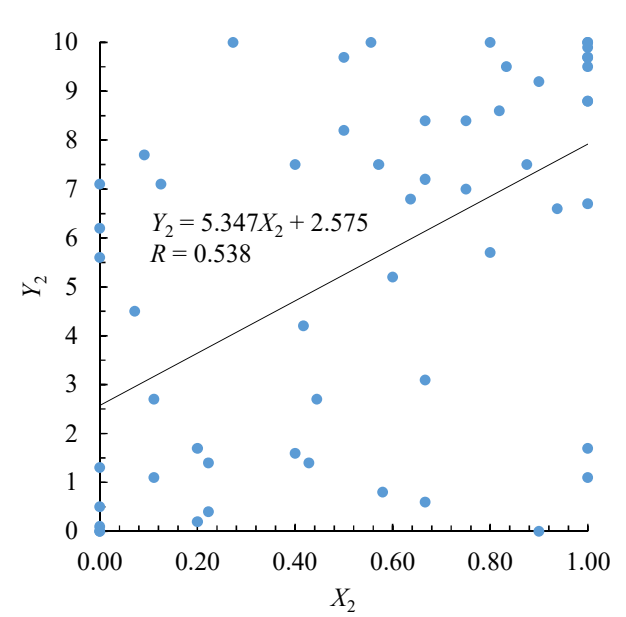

(b)

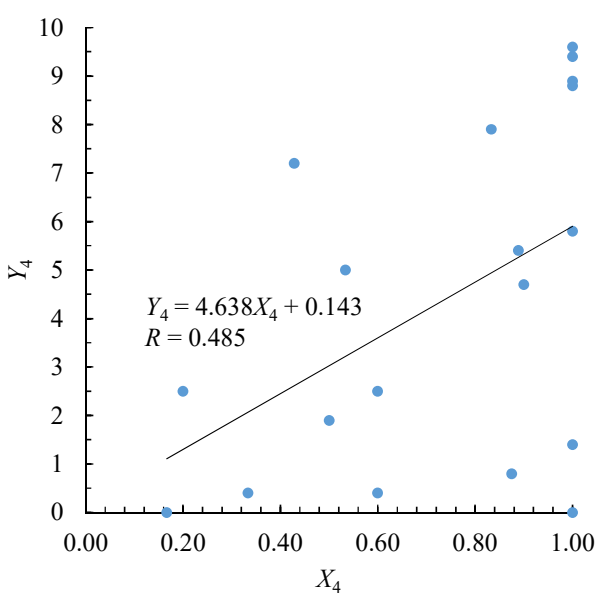

(d)

Figure 5. The relationship between the incidence rates of each of the profiles and the tactile score. (a) $X_{1}=$ incidence rates of the one-step increase pattern; $Y_{1}=$ score of the soft feel. (b) $X_{2}=$ incidence rates of the two-step increase pattern; $Y_{2}=$ score of the hard feel. (c) $X_{3}=$ incidence rates of the double peak pattern; $Y_{3}=$ score of the sticky feel. (d) $X_{4}=$ incidence rates of the drastic decrease pattern; $Y_{4}=$ score of the snapped feel. 


\section{Discussion}

In this study, we simultaneously evaluated the psychological and physical data when test subjects grasped/cut selected objects. Here, we discuss some of the physical factors that determined the tactile feels. First, we examined the dominant factor leading to a sticky feel. Of the 17 subjects whose incidence rate of the double peak pattern was $80 \%$ or more, 14 scored the sticky feel at eight or greater. On the other hand, dry feel was perceived for the one-step increase pattern, two-step increase pattern, or drastic decrease pattern, in which $F_{x y}$ did not increase when the blade broke contact from the object. These results indicated that the sticky feel was caused by an abrupt increase and decrease of the force when the grasping tools separated from the objects. Second, we considered the mechanisms of hardness. Previous studies have shown that hardness is judged based on the distribution of the reaction force when a human touches an object directly with a finger [16,17]. How do we recognize hardness when we touch objects through tools, such as scissors? The incidence rates of the one-step increase pattern and two-step increase pattern showed positive correlations with soft and hard feels, respectively ( $R=0.541$ and 0.538 , respectively). In each of the processes, a few characteristic points were observed in the movement of the blades. The $\theta$ decreased $8.1 \pm 2.6^{\circ}$ in the grasping process of the urethane resin, which has a low elastic modulus. On the other hand, the $\theta$ only decreased $1.6 \pm 0.9^{\circ}$ when the stainless steel plate was grasped, which has a high elastic modulus. Hardness can be reflected as the ratio of the applied force and the deformation of objects. When subjects touch objects through tools, we might be able to determine the hardness based on the movement of blades or hands. We can recognize the hardness of an object if it leads to an instantaneous increase in the reaction force with only a small movement in the blades.

Finally, the mechanism of tactile feels that are perceived strongly in the cutting process is discussed. In the paper cutting process, dry, creaking, and hard feels were perceived strongly: The scores were $6.6 \pm 2.8,6.8 \pm 2.5$, and $8.2 \pm 2.7$, respectively. In the mechanical evaluation, the drastic decrease pattern was observed only when the subjects cut paper. In this profile, $F_{x y}$ fluctuated (amplitude of $0.07 \pm 0.05 \mathrm{~N}$ ) for $0.2 \mathrm{~s}$ when cutting the paper. This vibration was observed for all of the subjects and must contribute to the tactile sense of the cutting process. Many studies have reported that fluctuations in frictional resistance occur on the finger surface of humans and cause a stick-slip feeling [15,18-20]. Funahashi et al. reported that vibration feedback heightened the reality of virtual cutting [14]. It was suggested that dry, creaking, and hard feels were derived from fine fluctuations of $F_{x y}$.

During the evaluation, the subjects pinched the handles of the forceps and scissors, but this situation is different from the ordinary operation. Since the contact state between the objects and blades does not change even if the pinching situation changes, the force profiles are not affected. On the other hand, when the contact area between the finger and the scissors increases, more tactile information can be obtained and it may affect the tactile impression. Considering leverage, although the blade length affects the magnitude of the grasping and cutting force, the length does not affect the force profiles.

\section{Conclusions}

In the present study, we evaluated the tactile feels and grasping/cutting force when subjects grasp/cut some objects with forceps or scissors. Hardness was perceived by the change of force with small blade movements when the scissors were in sustained contact with the objects. Stickiness was perceived by the increase and decrease of force when the blades peeled off from the objects. The cutting sensation was affected by fine force fluctuations during the scissors closing, and a rapid decrease in force at the moment cutting was completed. Recently, many researchers have focused on the development of an electronic skin and tactile-based active object discrimination technologies [21-23]. The present findings will be useful for the design of virtual reality systems and surgical robots. To develop these fields, it is required to clarify the perception mechanisms of textures or shapes through scissors. 
Author Contributions: Conceptualization, Y.N., M.W. and Y.A.; Formal analysis, M.W. and Y.A.; Funding acquisition, Y.N. and J.N.; Investigation, M.W. and Y.A.; Writing—original draft, M.W.; Writing—review \& editing, Y.N.

Funding: This research was funded by next-generation robot core technology development of NEDO, Japan.

Conflicts of Interest: The authors declare no conflict of interest. The funders had no role in the design of the study; in the collection, analyses, or interpretation of data; in the writing of the manuscript, and in the decision to publish the results.

\section{References}

1. Haag, R.; Storz, W. Surgical Scissors. In Springer Handbook of Medical Technology, 2nd ed.; Kramme, R., Hoffmann, K.P., Pozos, R.S., Eds.; Springer Science \& Business Media: Berlin/Heidelberg, Germany, 2011; pp. 1291-1319. ISBN 978-3-540-74658-4.

2. Sergeeva, A.; Huysman, M.; Faraj, S. Transforming work practices of operating room teams: the case of the Da Vinci robot. In Proceedings of the 36th International Conference on Information Systems, Fort Worth, TX, USA, 20-22 September 2015; pp. 1-10.

3. Gandevia, S.C.; McCloskey, D.I. Changes in motor commands, as shown by changes in perceived heaviness, during partial curarization and peripheral anaesthesia in man. J. Physiol. 1997, 272, 673-689. [CrossRef]

4. Henningsen, H.; Ende-Henningsen, B.; Gordon, A.M. Contribution of tactile afferent information to the control of isometric finger forces. Exp. Brain Res. 1995, 105, 312-317. [CrossRef] [PubMed]

5. Jones, L.A.; Piateski, E. Contribution of tactile feedback from the hand to the perception of force. Exp. Brain Res. 2006, 168, 298-302. [CrossRef] [PubMed]

6. Mahvash, M.; Voo, L.; Kim, D.; Jeung, K.; Wainer, J.; Okamura, A. Modeling the forces of cutting with scissors. IEEE Trans. Biomed. Eng. 2008, 55, 848-856. [CrossRef] [PubMed]

7. Tanaka, A.; Hirota, K.; Kaneko, T. Representation of force during virtual cutting operation. EMNLP. Trans. IPS. Japan. 1999, 40, 2978-2987.

8. Greenish, S.; Hayward, V.; Chial, V.; Okamura, A.; Steffen, T. Measurement, analysis, and display of haptic signals during surgical cutting. Presence 2002, 11, 626-651. [CrossRef]

9. Mahvash, M.; Hayward, V. Haptic rendering of cutting: A fracture mechanics approach. Available online: https: / / digital.lib.washington.edu/researchworks/bitstream/handle/1773/34885/he-v2n3.pdf? sequence $=1 \&$ isAllowed $=y$ (accessed on 11 July 2018).

10. Okamura, A.M.; Webster III, R.J.; Nolin, J.T.; Johnson, K.W.; Jafry, H. The haptic scissors: Cutting in virtual environments. In Proceedings of the 2003 IEEE International Conference on Robotics and Automation, Taipei, Taiwan, 14-19 September 2003; Volume 3, pp. 828-833.

11. Fujino, S.; Sato, D.; Abe, K.; Konno, A.; Uchiyama, M. Displaying feeling of cutting by a micro-scissors type haptic device. In Proceedings of the 2008 IEEE International Conference on Robotics and Automation, Pasadena, CA, USA, 19-23 May 2008; pp. 2067-2072.

12. Weiss, D.J.; Okamura, A.M. Haptic rendering of tissue cutting with scissors. In Proceedings of the 12th Annual Medicine Meets Virtual Reality Conference, Newport Beach, CA, USA; 2004; pp. 407-409.

13. Fratu, A.; Fratu, M. Analytical model of the cutting process with scissors-robot for haptic simulation. Bull. Transylv. Univ. Braşov, Ser. I 2011, 4, 113-120.

14. Funahashi, K.; Kubotani, D.; Iwahori, Y.; Tanida, K. Virtual scissors in a thin haptic and force feedback environment. JACIII 2009, 13, 283-288. [CrossRef]

15. Nonomura, Y.; Saito, R.; Takahashi, A. Friction at fingertip surface during water contact process. Bull. Chem. Soc. Jpn. 2015, 88, 949-954. [CrossRef]

16. Srinivasan, M.A.; Lamotte, R.H. Tactual discrimination of softness. J. Neurophysiol. 1995, 73, 88-101. [CrossRef] [PubMed]

17. Fujita, K.; Ohmori, H. A new softness display interface by dynamic fingertip contact area control. In Proceedings of the 5th World Multiconference on Systemics, Cybernetics and Informatics, Orlando, FL, USA, 22-25 July 2001; pp. 78-82.

18. Nonomura, Y.; Arashi, Y.; Maeno, T. How do we recognize water and oil through our tactile sense? Colloids Surf. 2009, 73, 80-83. [CrossRef] [PubMed] 
19. Nonomura, Y.; Fujii, T.; Arashi, Y.; Miura, T.; Maeno, T.; Tashiro, K.; Kamikawa, Y.; Monchi, R. Tactile impression and friction of water on human skin. Colloids Surf. 2009, 69, 264-267. [CrossRef] [PubMed]

20. Nonomura, Y.; Miura, T.; Miyashita, T.; Asao, Y.; Shirado, H.; Makino, Y.; Maeno, T. How to identify water from thickener aqueous solutions by touch. J. R. Soc. Interface 2011. [CrossRef] [PubMed]

21. Yogeswaran, N.; Dang, W.; Navaraj, W.T.; Shakthivel, D.; Khan, S.; Polat, E.O.; Gupta, S.; Heidari, H.; Kaboli, M.; Lorenzelli, L.; et al. New materials and advances in making electronic skin for interactive robots. Adv. Robot. 2015, 29, 1359-1373. [CrossRef]

22. Kaboli, M.; Yao, K.; Feng, D.; Cheng, G. Tactile-based active object discrimination and target object search in an unknown workspace. Auton. Robots 2018, 1-30. [CrossRef]

23. Feng, D.; Kaboli, M.; Cheng, G. Active prior tactile knowledge transfer for learning tactual properties of new objects. Sensors 2018, 18, 634. [CrossRef] [PubMed]

(C) 2018 by the authors. Licensee MDPI, Basel, Switzerland. This article is an open access article distributed under the terms and conditions of the Creative Commons Attribution (CC BY) license (http:/ / creativecommons.org/licenses/by/4.0/). 